\title{
Gradient-Based Routing in Sensor Networks
}

\author{
Jabed Faruque Ahmed Helmy \\ Department of Computer Engineering \\ University of Southern California, Los Angeles, CA 90089, USA \\ (faruque, helmy)@usc.edu
}

\begin{abstract}
A distributed sensor network (DSN) consists of sensor nodes with limited energy source, sensor devices, short-range radio and on-board processing capability. Sensing capability of the attached sensing devices and their small size, make these sensor nodes highly suitable for environmental monitoring. But characteristics of sensor nodes, e.g., expensive wireless communication, high probability of failure or malfunction and the unstructured nature of DSN, make routing in DSN a challenging problem. Traditional routing protocols in DSN are based mostly on flooding (Directed Diffusion[1]) or random-walk (Rumor routing[1], ACQUIRE [3], etc.). Flooding causes huge communication overhead due to frequent route discovery, especially for one-shot query and for large-scale networks. Though asymptotically, random-walk shows good performance, in practice it causes high latency and without directionality and proper TTL, sometimes fails to find resource. We note that many physical phenomena follow decay law. For example, if some location's temperature is $100^{\circ} \mathrm{C}$, then nearby locations temperature should be correlated with that based on distance. So, routing protocols can use this natural gradient as an important attribute to forward the query towards source. A proactive informative driven protocol is proposed in [4] for querying and routing in sensor net. It uses proactive information utility measurement to select optimal node and guide query routing, which creates extra overhead at low query rate due to exchange of information between neighbors and leader. Also that protocol does not handle scenarios with local maxima or minima or multiple sources. To overcome these limitations, in this paper we propose a novel energy efficient, fully distributed and reactive routing protocol based on gradient of event's effect for environmental monitoring sensor networks. Our protocol handles local and isolated maxima or hole of information using simulated annealing concepts.
\end{abstract}

In practice, sensor devices are not perfect. Theoretically, the tail of the decay distribution is infinite, but real life sensors are unable to detect or measure effect of an event below a certain threshold. So, some flat information region always exists, where information content is either the same or zero due to lack of sensitivity of sensor device(s). Also, obstacles or sensor/node failure may cause discontinuity in the decay distribution (Figure 1).

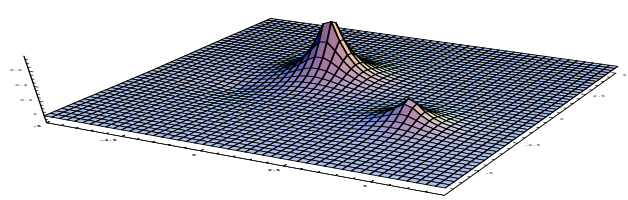

Figure 1: Decay curve with local maxima and hole

In our protocol a query (about an event) is forwarded based on the information gradient in the sensor nodes. A node forwarding the query to its neighbors includes its information level about the queried event. Each neighbor decides whether to forward the query based on the algorithm described below. If a node is able to resolve the query, it uses the reverse path to reply. Otherwise, our routing algorithm has three parts:

1. In the decay region (in which the information gradient is not zero), we follow a greedy approach. If a node is able to improve information level, it forwards the query to its neighbors for further improvement. Otherwise, the node performs probabilistic forwarding, described in 2.

2. The type of discontinuity in the smooth decay curve can be sharp drop or rise of information level about the queried event due to sensing errors. To handle such local and isolated maxima or hole of information, we use a probabilistic forwarding function $\left(p f(x)=\frac{1}{x^{a}}\right.$, where $x=$ hop-count), based on the simulated annealing concept. 
3. To forward a query in the flat information region (in which the information gradient is zero) instead of flooding, we use multiple random walks. If good and wrong moves towards decay region are equally likely and each node forwards the query to half of its neighbor(s), we can show that for each node, $\operatorname{Pr}$ [wrong move] $=\prod_{i=1}^{\frac{n}{2}} \frac{\frac{n}{2}-i+1}{n-i+1}$. From Figure 2, it is clear that the probability of failure to reach the decay region is negligible for $n \geq 6$, where $n=$ number of neighbors. In the absence of events, this part will handle the query.

Initially our algorithm generates multiple paths to discover source; but in the absence of multiple sources most of the paths will terminate after few hops.

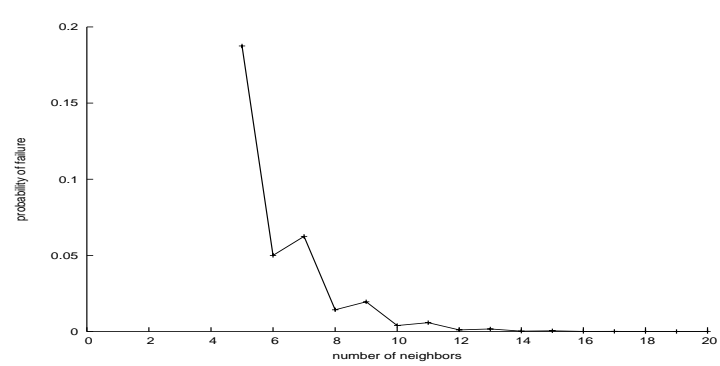

Figure 2: Probability of failure vs number of neighbors

To evaluate the protocol, simulations were conducted with $100 \times 100$ grid topology. Each node can communicate with its eight neighbors. In our primary experiment setup, we consider one peak information point, located at node 0 and the querier can be any node. Effect of an event follows the decay law $\left(f(d) \propto \frac{1}{d^{\alpha}}\right.$, where $d=$ distance from peak, and $\alpha$ is the decay parameter depending on type of effect). In the current state of our work, all experiments are done in the decay region (with hole and local maxima). The integration of flat region is in progress.

Through simulations we analyzed the average communication cost in term of average number of transmissions, and compared it with flooding and expending ring search (ERS) in Figure 3. Reachability of the protocol is analyzed for the different

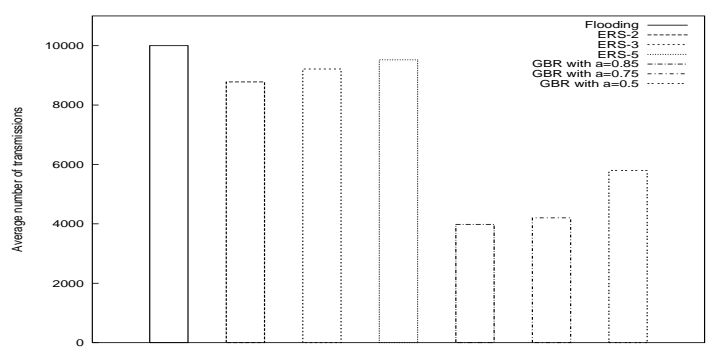

Figure 3: Average energy dissipation by Flooding, ERS and Gradient Based Routing (GBR) values of $a$ of the probabilistic forwarding function. From Figures 4 and 5 we see that for $a<\alpha$, the query failure rate is very low. But, it increases the number of transmissions as more nodes

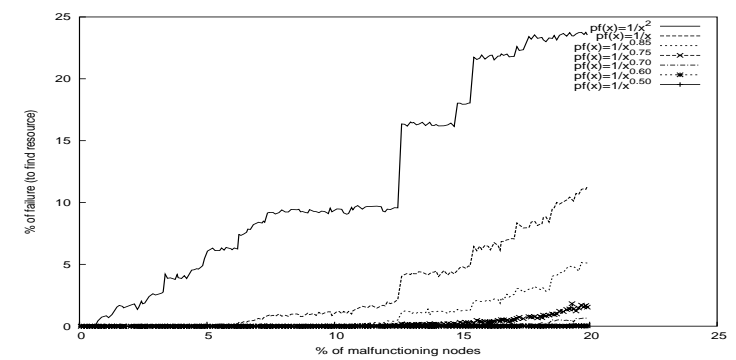

Figure 4: Percentage of failed queries vs percentage of malfunctioning nodes

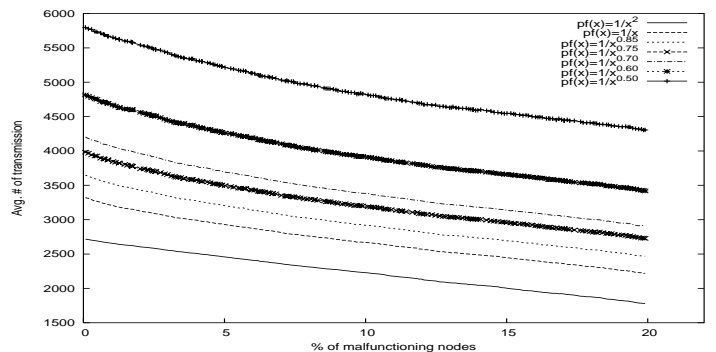

Figure 5: Average number of transmissions vs percentage of malfunctioning nodes

are selected to forward the query probabilistically. However, for values of $a<\alpha$ but close to $\alpha$ we get the optimal trade-off between communication overhead and robustness of the protocol.

In sum, the main objective of our protocol is to $e x$ ploit the effect decay pattern of events to efficiently route the query towards the event source. Also it can be used to detect boundary of an event by using edge detection logic proposed in [5] as a gradient. Using time gradient, the protocol can be used for tracking moving object (e.g. animal or vehicle). Currently we are working to further reduce the number transmissions and enhance reachablity, based on an analytical relationship between the decay pattern and the probabilistic forward function at each step. Also we are studying the behavior of the protocol with multiple sources and are comparing the performance of our protocol with that proposed in [4].

\section{References}

[1] C. Intanagonwiwat, R. Govindan and D. Estrin, Directed Diffusion: A Scalable and Robust Communication Paradigm for Sensor Networks, MobiCom 2000.

[2] D. Braginsky and D. Estrin, "Rumor Routing Algorithm for Sensor Networks", WSNA '02, September 28, 2002, pp 22-31.

[3] N. Sadagopan, B. Krishnamachari, and A. Helmy, "Active Query Forwarding in Sensor Networks (ACQUIRE)", SNPA '03, May 2003.

[4] M. Chu, H. Haussecker, and F. Zhao, "Scalable Information-Driven Sensor Querying and Routing for ad hoc Heterogeneous Sensor Networks" Int'l J. High Performance Computing Applications, 16(3):90110, Fall 2002.

[5] K. Chintalapudi, R. Govindan, "Localized Edge Detection in Sensor Fields", Ad-hoc Networks Journal, 2003. 